\title{
Upgrading the OVRO-LWA for improved cosmic ray detection capabilities
}

\author{
Kathryn Plant* \\ Department of Astronomy, California Institute of Technology, Pasadena, California, USA \\ E-mail: kpeastro.caltech.edu
}

Gregg Hallinan ${ }^{1}$, Andres Romero-Wolf ${ }^{2}$, Ryan Monroe ${ }^{1}$, Jonathon Kocz ${ }^{1}$, Anna Nelles $^{3,4}$, Marin Anderson ${ }^{1}$, Michael Eastwood ${ }^{1}$, Larry D'Addario ${ }^{1}$, David Woody ${ }^{5}$, James Lamb ${ }^{5}$, Washington Carvalho $\mathrm{Jr}^{6}{ }^{6}$, Konstantin Belov ${ }^{2}$

${ }^{1}$ Department of Astronomy, California Institute of Technology, Pasadena, California, USA; ${ }^{2}$ Jet Propulsion Laboratory, California Institute of Technology, Pasadena, California, USA; ${ }^{3}$ Institut für Physik, Humboldt-Universität zu Berlin, Germany; ${ }^{4}$ DESY, Platanenalle 6, 15738 Zeuthen, Germany; ${ }^{5}$ California Institute of Technology, Owens Valley Radio Observatory, Big Pine, California, USA; ${ }^{6}$ Departamento de Física, Universidade de São Paulo, São Paulo, Brazil;

The Owens Valley Long Wavelength Array has made self-triggered radio detections of cosmic-ray air showers (Monroe et al. 2019 submitted, see Romero-Wolf et al. proceedings PoS(ICRC2019)405) using 256 irregularly-spaced dual-polarization dipole antennas with $\sim 60 \mathrm{MHz}$ bandwidth centered around $55 \mathrm{MHz}$ and maximum antenna separations of $1.5 \mathrm{~km}$. Over the next two years, an upgrade to 352 antennas up to $2.6 \mathrm{~km}$ apart and new signal processing infrastructure will expand the capabilities of the array, including its sensitivity to cosmic ray air showers. In addition to the benefit of a larger area, wider antenna separations will improve the sensitivity to inclined events, thus increasing the detection rate of higher-energy cosmic rays. Furthermore, an observing mode with hierarchical beam-forming (grouping subsets of dipoles in-phase) will increase the detection rate of lower-energy cosmic rays. Detecting air showers requires response to ten-nanosecond-timescale signals, coincidenced across the array, and thus requires novel FPGA firmware. This poster will focus on the preliminary design of the new digital signal processing system.

36th International Cosmic Ray Conference -ICRC2019-

July 24th - August 1st, 2019

Madison, WI, U.S.A.

${ }^{*}$ Speaker. 


\section{Introduction}

Monroe et al. (submitted 2019) accomplished an RF-only detection of cosmic rays with the current Owens Valley Radio Observatory Long Wavelength Array (OVRO-LWA) in a dedicated observing run (see Romero-Wolf et al., proceedings PoS(ICRC2019)405). On the upgraded 352element array, a cosmic ray detection system will run fully commensally alongside the other observing modes of the array (including beamforming and all sky imaging).

The OVRO-LWA will be sensitive to airshowers from cosmic rays with energies in the range $3 \times 10^{16} \mathrm{eV}-10^{18} \mathrm{eV}$, probing the transition from Galactic to extragalactic cosmic rays. The radio pulse arrival times and intensities at individual antennas across the array will be used to reconstruct the column depth, $X_{\max }$, of the maximum number of shower particles in the event, which is sensitive to characteristics of the initial high energy cosmic ray particle. See (Carvalho et al., proceedings PoS(ICRC2019)211) for more about $X_{\max }$ reconstruction for the OVRO-LWA. The OVRO-LWA is sky-noise limited by the Galactic background (rather than receiver-noise limited) throughout its band.

With 352 dual-polarization antennas sampled at $200 \mathrm{MHz}$, the full array will produce data at $140.8 \mathrm{~GB} / \mathrm{s}$, which is far more data than can be sent off the FPGAs to CPUs for processing. Thus, observing cosmic ray radio airshowers without an external trigger means that events must be detected on the FPGAs themselves in order to trigger buffered data to be sent to CPUs.

Section 2 describes the airshower detection technique and the planned firmware to trigger sending buffered data for candidate events. Section 3 briefly describes the ADC and FPGA boards that will be used in the upgraded OVRO-LWA. Section 4 discusses the expected event rates and the software pipeline. Section 5 discusses options for leveraging antenna groupings to flag RFI.

\section{Detection technique and Firmware}

As an airshower wavefront passes across the array, each antenna within the shower footprint will be illuminated with a impulsive transient. The timescale of the bandlimited impulse will be $20 \mathrm{~ns}$ for this instrument, and in order to detect such fast events the detection must take place on the FPGA. Figures 1 and 2 overlay simulated airshowers (see Carvalho et al. proceedings PoS(ICRC2019)211) for a $5 \times 10^{17} \mathrm{eV}$ proton over a plot of the proposed antenna positions of the upgraded array. Figure 1 shows a simulated event arriving at the north, inclined at 60 degrees and Figure 2 shows an event from zenith arriving on the close-packed core antennas of the array. 


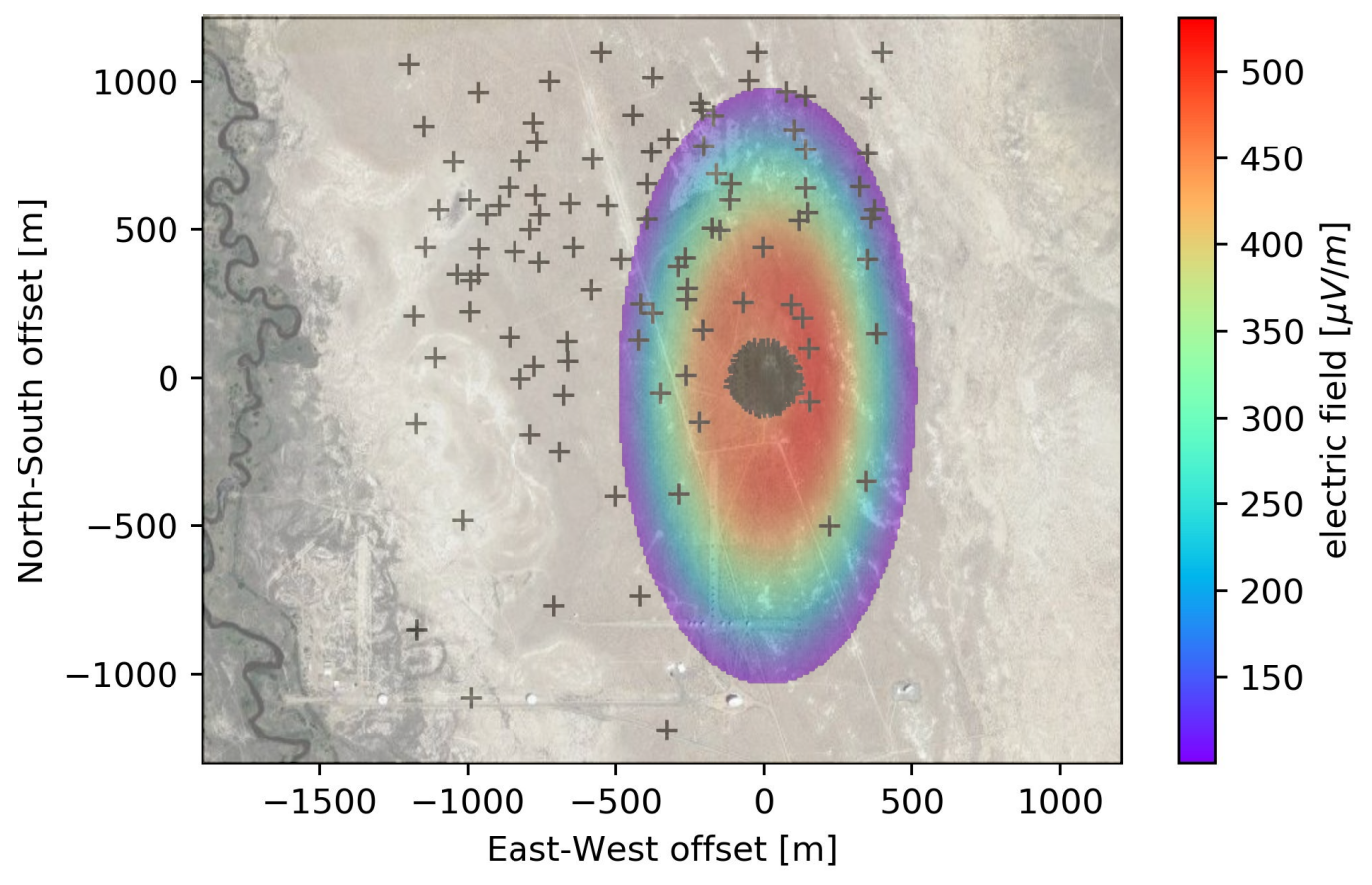

Figure 1: The colormap shows the peak electric field during a simulated airshower for a $5 \times 10^{17} \mathrm{eV}$ cosmic ray proton arriving from the North and inclined at 60 degrees. The colormap is only plotted for regions where the peak electric field would be detectable by an OVRO-LWA antenna. The black markers show the proposed positions of antennas in the upgraded OVRO-LWA. The black circle in the core of the array is due to the density of antenna markers. The background is a satellite image (source: Google maps) of the observatory.

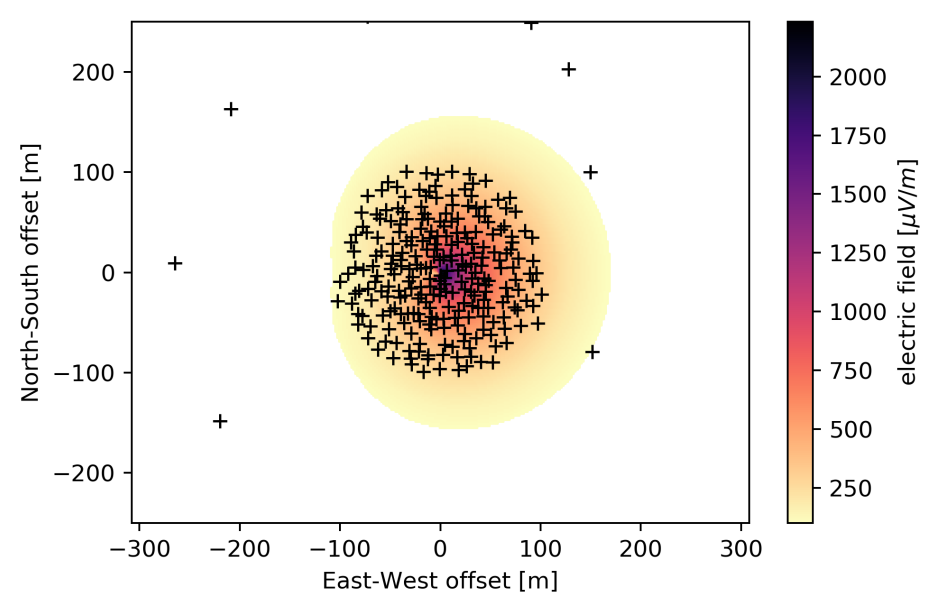

Figure 2: The colormap shows the peak electric field during a simulated airshower for a $5 \times 10^{17} \mathrm{eV}$ cosmic ray proton arriving from zenith and centered on the central core of the array. The colormap is only plotted for regions where the peak electric field would be detectable by an OVRO-LWA antenna. The black markers show the proposed positions of antennas near the dense central core of the upgraded OVRO-LWA. 
Figure 3 outlines the different types of information flow required by an FPGA-based radioonly trigger system. Trigger signals will be communicated between FPGAs, raw voltage data for detected candidates is transmitted from the FPGAs to the CPUs, and the CPUs transmit system configuration information (i.e. thresholds and cable delays) to software registers on the FPGAs.

Each FPGA processes a subset of the array's signals (64 inputs per board for a SNAP2, see section 3) and must decide when to trigger the whole array to save data based on that local subset of signals. While buffering 4096 raw ADC samples in FPGA block RAM (BRAM), a parallel stream of voltages is processed in FPGA logic to detect pulse peaks in each antenna's data stream and then count the number of coincident detections to determine that a candidate impulsive wavefront is passing across the array (all-sky search). Running in parallel, we will check for events from known RFI directions by delaying the data streams according to the specific direction to veto. If a detection in the all-sky search doesn't coincide with a veto from the RFI blockers, a trigger message causes the buffered voltages to be sent over ethernet to CPUs. The trigger will also be sent to all the other FPGAs, so that they send their buffered voltages for the event.

Figures 4 and 5 outline this firmware. To detect an impulse in a single antenna's voltage stream, a thresholding block outputs Boolean 1 when the input power exceeds a threshold. The Boolean output stream is 1 for exactly one clock cycle, and immediate repeats are blocked for a fixed window of time, as part of the RFI rejection strategy. For each single-antenna signal, the Boolean 1 pulse is then extended for the number of time samples corresponding to the maximum possible geometric delay across the array. This resulting data stream is then summed over all the antennas on the FPGA. If the summed count is above a set threshold, then the group of antennas on that FPGA received pulses within the arrival times consistent with an impulsive event sweeping across the array. If this event doesn't coincide with a veto from the direction-based RFI blockers, then a trigger will be transmitted and the buffered data from the whole array will be saved. The RFI blockers are a similar process except that the pulses are not extended for the light travel time across the array, and instead the streams are delayed according to a specific direction of arrival, prior to summing over the different antennas.

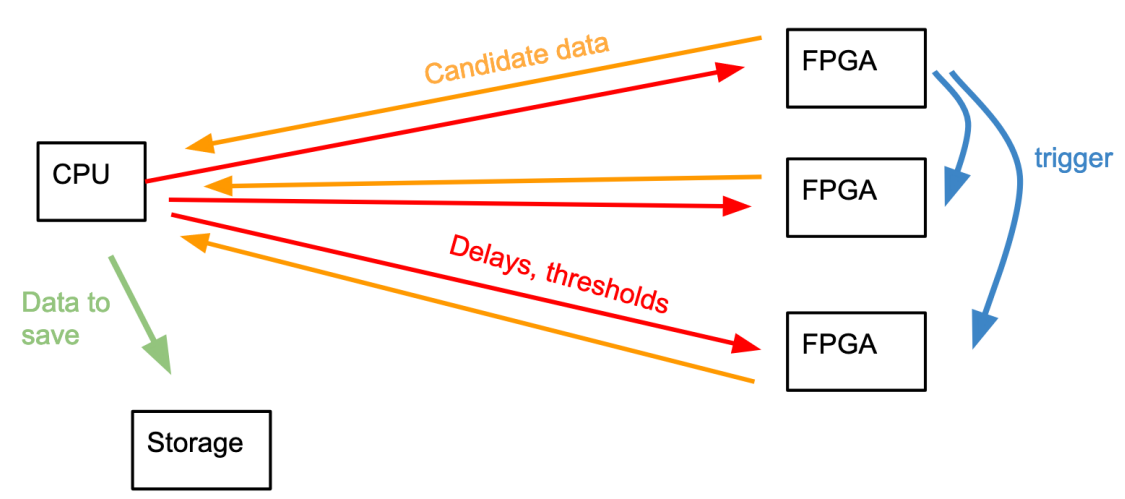

Figure 3: System overview. Delays and thresholds are sent to the FPGAs from the CPUs. Triggers are communicated among FPGAs. Buffered data for candidate events is transmitted from FPGAs to CPUs. 


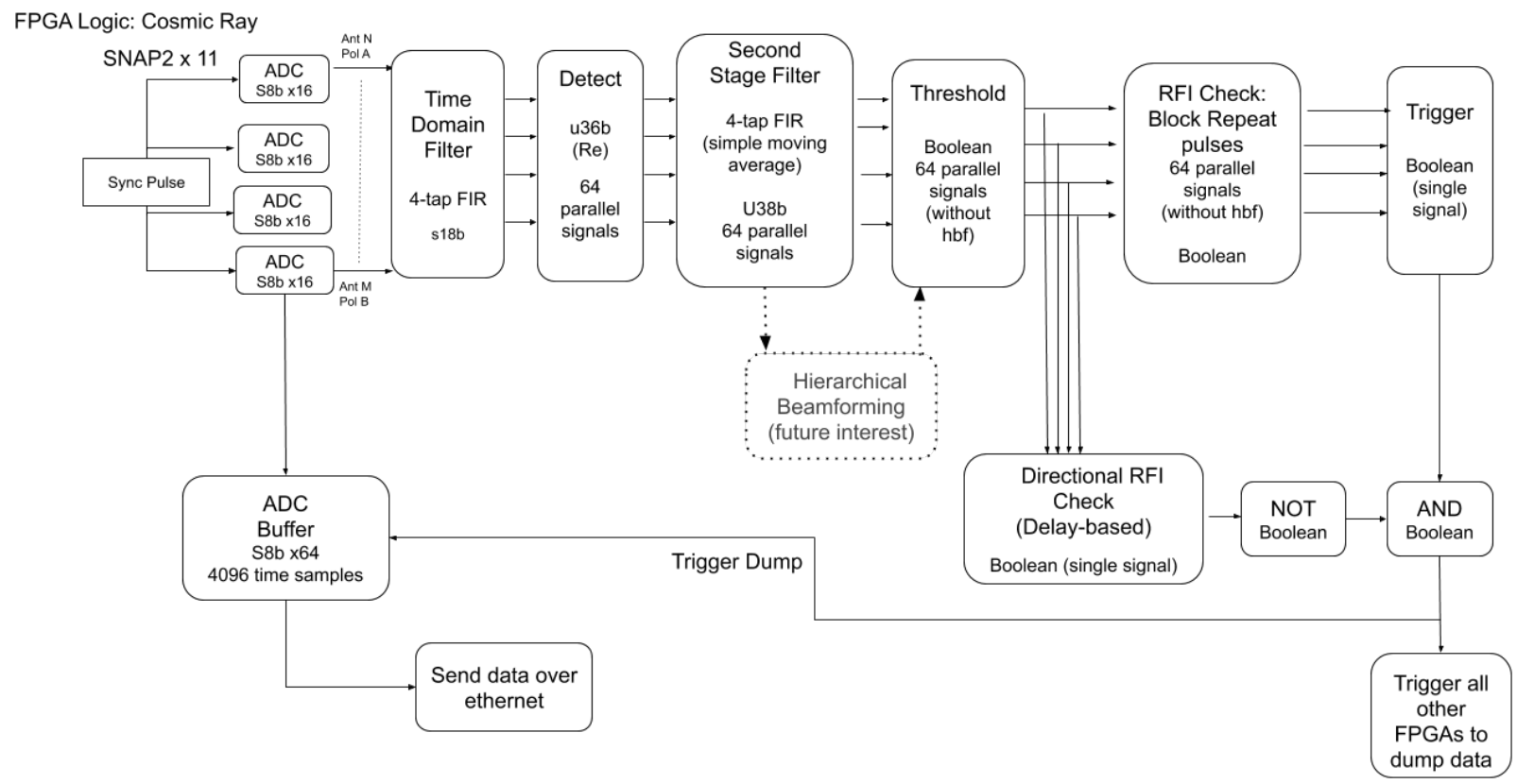

Figure 4: Firmware overview.

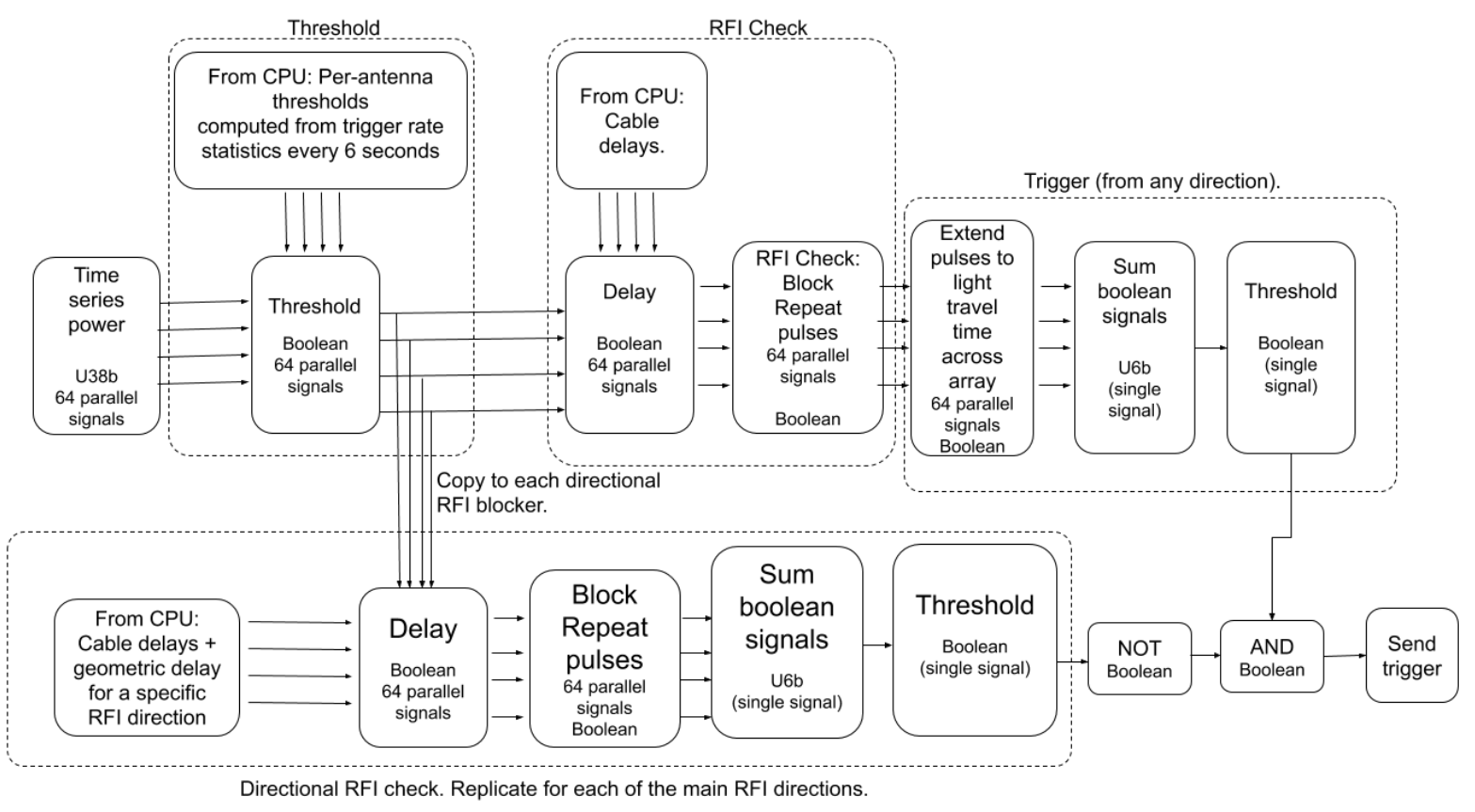

Figure 5: Detection and trigger detail. 
Preliminary firmware has been compiled using a SNAP1 board to estimate resource use (BRAM, logic, IO, etc). BRAM will be the most limited resource on the FPGA. Scaling for the number of inputs and additional resources on the SNAP2 compared to the SNAP1, the expected BRAM use is about $10 \mathrm{MB}$, or $13 \%$ of the SNAP2 available BRAM. This will fit alongside the signal processing for the other science cases.

The cosmic ray system's main use of BRAM is in the voltage buffer. Since much of the trigger process uses Boolean signals, resource use is small in this part of the system. Firmware was compiled with and without a directional RFI blocker to demonstrate that a Boolean directional flagger uses very little additional resources.

\section{Electronics}

In the upgraded array, the cosmic ray detection system will be operating throughout regular observing alongside the other science programs on the array. The digital electronics will be fully upgraded and we plan to use 11 SNAP2 FPGA boards. The choice to use SNAP2 boards is partly motivated by the size of the FPGA, which is large enough to simultaneously run the different signal processing required by the different science cases, and the number of input signals that can be processed in parallel by a single board. A SNAP2 board has a Xilinx Kintex Ultrascale (XCKU115) FPGA, an ARM auxiliary processor, and four $40 \mathrm{~Gb}$ ethernet ports.

We plan for each SNAP2 to supervise four 16-input ADC cards. The ROACH2 boards currently in use process 16 input signals per board. Having 64 signals per board in the new system is particularly useful for the cosmic ray science case because the initial detection is based on the subsets of antennas processed by individual boards. Firmware will be developed using the CASPER toolflow and libraries (see e.g. Hickish et al. 2016), which make it possible to rapidly create firmware for radio astronomy signal processing applications.

\section{Expected Event Rates}

Data processing for the OVRO-LWA cosmic ray experiment takes place in three main stageson-FPGA event detection, software signal processing of candidates, and final analysis of promising candidates.

For 352 dual polarization antennas and 4096 time samples, each candidate is 2.883584 MBytes. Based on Monroe et al. (2019 submitted) we expect 40 triggers per second, which is an average data rate of 115.3 MBytes per second. The candidate rate at each stage in the data processing is summarised in table 1 .

After packet capture, the raw ADC voltages will be re-processed by a CPU with filters and power detection similar to the first stages of processing in the FPGA in figure 2. Then, a pulse arrival time (TOA), a peak power, and noise power will be computed for each antenna. A wavefront model is fit to the TOA at each antenna position, to test whether the candidate event is described by a wavefront moving across the array. Events with a large residual from the TOA fit are removed from the sample, thus rejecting events caused by thermal noise in the individual antennas. 


\begin{tabular}{|c|c|c|c|c|}
\hline Processing step & $\begin{array}{c}\text { Reduces events } \\
\text { by factor of }\end{array}$ & $\begin{array}{c}\text { Number of } \\
\text { events daily }\end{array}$ & hardware & data rate \\
\hline block repeat events & 10 & $3.6 \times 10^{7}$ & FPGA & $1.25 \mathrm{~GB} / \mathrm{s}$ \\
block specific directions & 10 & $3.6 \times 10^{6}$ & FPGA & $125 \mathrm{MB} / \mathrm{s}$ \\
wavefront fit & 3 & $1.2 \times 10^{6}$ & software & $41.7 \mathrm{MB} / \mathrm{s}$ \\
further direction-based flagging & 1000 & 1200 & software & $41.7 \mathrm{kB} / \mathrm{s}$ \\
airplane rejection & 100 & 12 & analysis pipeline & $416.7 \mathrm{bytes} / \mathrm{s}$ \\
polarization and spatial power & 2 & 6 & analysis pipeline & 208.3 bytes/s \\
\hline
\end{tabular}

Table 1: Candidate processing steps. Rate predictions approximated from observations in Monroe et al. 2019 submitted

For all the events that are well-fit by a wavefront, the fit has thus assigned the event a source direction (and distance, in the case of near-field RFI). Most events remaining at this stage will be clustered at known locations of RFI sources. Based on Monroe et al. (2019 submitted), rejecting events from known RFI locations reduces the sample size by a predicted factor of 10000 . We estimate that a factor of 10 will be achieved on the FPGA, leaving a factor of 1000 to software processing. In Monroe et al. 99\% of the remaining events trace airplane flight paths. After rejecting these reflections off airplanes, one candidate per few hours is expected to remain.

Polarization and spatial power distribution will be used as the final criteria for identifying cosmic rays. Monroe et al. compared power in the North-South dipole to power in the East-West dipole to identify cosmic rays. The upgraded system plans to compute a full stokes vector for each of the events remaining at this stage in the pipeline. The geomagnetic component of cosmic ray emission is linearly polarized according to the earth's field lines. The (usually smaller) Askaryan component is linearly polarized in the direction radial to the axis of the shower. Most events are expected to be dominated by the geomagnetic component, but this will be weaker for events arriving to the south of the array. Thus, computing full Stokes information and comparing to the arrival direction will allow us to better characterize these events. The spatial distribution of emission will be compared to models of cosmic ray airshower footprints.

\section{Antenna Grouping to FPGAs}

In the current 288-element OVRO-LWA (of which Monroe et al. (2019) used the 256 core antennas), antennas are grouped to FPGAs in sets of 16 arranged in North-South stripes. Since cosmic ray detection occurs on the FPGAs, direction-based RFI flagging can be improved by careful choice of the antenna groups processed by each FPGA. Due to the location of the nearby town of Bishop and the orientation of the Earth's magnetic field lines, more RFI as well as more cosmic rays are expected to arrive from North of the array. Thus in the 352 element array, FPGA groups (64 signal inputs per board) will be chosen to allow precise directional sensitivity, to flag events from very specific RFI locations in the north with minimal loss of field of view toward the northern sky.

Since cables from all antennas are brought to one central location, there is no additional cost to choosing the order in which the cables are plugged in. Longer baselines (longer separations between pairs of antennas) make delay-based directional filtering more precise. However, clustering groups of nearby antennas can improve sensitivity by allowing heirarchical beamforming. We 
are currently investigating which antenna groupings optimize the balance between sensitivity and directional RFI blocking.

\section{Summary and Conclusions}

Monroe et al (2019 submitted) detected 10 cosmic rays in 40 hours. Scaling from this prototype, with one year of observing we expect to detect 2000 events with which to study the composition of cosmic rays in the $3 \times 10^{16} \mathrm{eV}-10^{18} \mathrm{eV}$ energy range. Detecting these events with a radio-only trigger at the OVRO-LWA requires novel FPGA firmware in order to identify candidates among the 140GB/s ADC output timeseries. This cosmic ray detection system will be designed to run commensally alongside the other observing modes of the array, which include beamforming and imaging for experiments in fields ranging from the epoch of reionization to extrasolar space weather.

\section{Acknowledgements}

This material is based in part upon work supported by the National Science Foundation under Grant AST- 1654815 and AST-1212226. The OVRO-LWA project was initiated through the kind donation of Deborah Castleman and Harold Rosen. K.P. is supported by a National Science Foundation Graduate Research Fellowship. W.R.C. thanks grants \#2015/15735-1 and \#2018/18876-3, SÃčo Paulo Research Foundation (FAPESP).

\section{References}

[1] R. Monroe et al. 2019, submitted for publication to Nucl. Instrum. Meth. A.

[2] R. Monroe, PhD. thesis, Caltech, 2018

[3] Hickish et al. 2016, JAI, Volume 5, Issue 4, id. 1641001-12 